\title{
Resonant Andreev reflection in a normal-metal-quantum-dot-superconductor system
}

\author{
Qing-feng Sun \\ Department of Physics, The University of Hong Kong, Pokfulam Road, Hong Kong, China \\ and Department of Physics, Peking University, Beijing 100871, China \\ Jian Wang \\ Department of Physics, The University of Hong Kong, Pokfulam Road, Hong Kong, China \\ Tsung-han Lin* \\ Department of Physics, The University of Hong Kong, Pokfulam Road, Hong Kong, China \\ and Department of Physics, Peking University, Beijing 100871, China \\ (Received 27 May 1998; revised manuscript received 25 August 1998)
}

\begin{abstract}
We investigate the electron tunneling through a normal-metal-quantum-dot-superconductor (N-QD-S) system where multiple discrete levels of the QD are considered. By using the nonequilibrium-Green's-function method, the current $I$ and the probability of the Andreev reflection $T^{A}(\omega)$ are derived and studied in detail. In addition to the resonant behavior of the Andreev tunneling as obtained in previous works, we find that the current $I$ versus the gate voltage $v_{g}$ exhibits different kinds of peaks, depending on the bias voltage, the level spacing of the QD, and the energy gap of the superconducting electrode. Besides, in $I-V$ characteristics extra peaks superimposed on the conventional current plateaus emerge, which stem from the resonant Andreev reflections. In the case with strongly asymmetric barriers, the BCS spectral density can be obtained directly from the $I-V$ characteristics. [S0163-1829(99)00405-1]
\end{abstract}

\section{INTRODUCTION}

In recent years there has been increasing interest in studying the transport properties of mesoscopic "hybrid" normalmetal-superconductor systems. The interplay of basic features from both of mesoscopics and superconductivity makes this subdiscipline of condensed-matter physics a very fruitful research field. ${ }^{1-3}$ Many works have been done on the subgap structures of the S-I-S or S-N-S junctions due to the effects of multiple Andreev reflections, ${ }^{4,5}$ the quantization of the maximum supercurrent of the superconducting quantum point contacts, ${ }^{6-9}$ the "zero-bias anomaly" of the S-I-N junctions, ${ }^{10-12}$ etc. The electron resonant tunneling through a quantum-dot (QD), either superconducting SQD or normal NQD, connected to two electrodes is another subject investigated extensively, including a variety of hybrid structures such as S-SQD-S, N-SQD-N, S-NQD-S, N-NQD-S, etc. For a small superconducting island with the energy gap greater than the Coulomb charging energy, the even-odd number effect produces a $2 e$ periodicity in the tunneling current versus gate-induced charge in S-SQD-S and N-SQD-N systems. ${ }^{13-18}$ Ralph, Black, and Tinkham measured the $I-V$ characteristics for a S-NQD-S from which the density of states of the quasiparticles in the superconducting leads has been obtained. ${ }^{19}$ A similar system has been studied theoretically by Yeyati et al. in the weak-coupling regime with asymmetric barriers by using the nonequilibrium-Green'sfunction (NGF) technique. ${ }^{20}$ Ishizaka, Sone, and Ando investigated the Kondo effect in a S-NQD-S system by the noncrossing approximation and obtained some interesting results. ${ }^{21}$ As for the N-NQD-S structures (simply by N-QD-S hereafter), Beenakker presented a general multichannel $S$-matrix description and predicted the resonant Andreev tun- neling for a single-level QD in the zero-bias limit. ${ }^{22}$ Beenakker's theory has been extended by Claughton, Leadbeater, and Lambert to the finite bias case and they found that the differential conductance resonances are strongly suppressed in the weak-coupling limit. ${ }^{3}$ Very recently, Fazio and Raimondi investigated the resonant Andreev tunneling through a strongly interacting QD in a N-QD-S structure, discussed how the Kondo effect can influence the two-particle tunneling, and obtained an enhancement of the Kondo anomaly in the $I-V$ curves due to the presence of a superconducting electrode. ${ }^{23}$

In this work, we shall investigate the electron tunneling through a N-QD-S structure, i.e., a normal quantum-dot connected to two leads, one is a normal metal and the other is a superconductor. Different from Refs. 3 and 22 where only a single level of the QD has been considered, here we assume that the QD has discrete multiple levels, but the intradot Coulomb interactions will be neglected for simplicity. Using the nonequilibrium-Green's-function (NGF) method, the current $I$ and the probability of the Andreev reflection $T^{A}(\omega)$ are derived which satisfied the gauge-invariant condition, i.e., the current remains unchanged when the potential (voltage) everywhere is changed by the same amount. ${ }^{24}$ The dependence of $I$ and $T^{A}(\omega)$ upon the gate voltage, the bias voltage, the level spacing of the QD, and the energy gap of the superconducting electrode have also been studied in detail. For this simple model system, we found very interesting results:

(1) Different from the N-QD-N system where the current $I$ or the differential conductance $d I / d V$ versus the gateinduced charge $Q\left(Q=C_{g} v_{g}\right)$ (or the gate voltage $\left.v_{g}\right)$ exhibits a series of peaks with equal interval, for the N-QD-S, the current $I$ versus $Q$ (or $v_{g}$ ) at small fixed bias $V$ exhibits 
more complicated pattern, which may have several different kinds of peaks with the same equal spacing for each kind of the peaks. This property originates from the multiple equalspacing levels of the QD we assumed. If the energy levels of the QD are not equal spacing, the series of peaks will be much more complicated.

(2) The resonant behavior can be seen either from $I$ vs $v_{g}$ or from the probability of Andreev reflection $(\mathrm{AR}), T^{A}(\omega)$. For a single N-S contact, the probability of the Andreev reflection strongly depends on the barrier height ${ }^{25}$ and is usually quite small for the realistic structures. ${ }^{4,5}$ However, in a $\mathrm{N}-\mathrm{QD}-\mathrm{S}$ system we considered, the probability of the Andreev reflection exhibits resonance under certain condition, although the transmission amplitudes of the left barrier and the right barrier are quite small. The two barriers between the QD and two leads play the role of two "mirrors" as in a Fabry-Perot interferometer.

(3) Extra peaks are found superimposed on the conventional current plateaus in $I-V$ curves due to the resonant Andreev reflections.

(4) Finally, for the case with strongly asymmetric barriers, the density of states of quasiparticles in superconducting electrode can be obtained directly from the $I-V$ characteristics. This result is similar to the experimental result by Ralph, Black, and Tinkham ${ }^{19}$ and the theoretical work by Yeyati et al. $^{20}$ for the S-NQD-S system.

The rest of this paper is organized as follows. In Sec. II we present the model and derive the gauge-invariant formula of the current $I$ and the probability of the Andreev reflection $T^{A}(\omega)$ by using the NGF technique. In Sec. III we study the properties of the current $I$ versus the gate voltage on the basis of the theoretical results obtained in Sec. II. We also discuss the resonant behavior and the different kinds of peaks of the resonant Andreev reflections which depend upon various factors such as the bias, the level spacing of the QD, and the energy gap of the superconducting electrode. The coefficient of the Andreev reflection is studied in Sec. IV. The properties of the $I-V$ curves and the density of states of the quasiparticles in the superconducting electrode are presented in Sec. V. Finally, a brief summary is given in Sec. VI.

\section{MODEL AND FORMULATION}

We assume that the system under consideration is described by the following Hamiltonian: ${ }^{9,21}$

$$
H(\tau)=H_{L}+H_{R}+H_{\mathrm{dot}}+H_{T}(\tau),
$$

where

$$
\begin{gathered}
H_{L}=\sum_{k, \sigma}\left(\epsilon_{L, k}^{0}-e v_{L}\right) a_{L, k \sigma}^{\dagger} a_{L, k \sigma}, \\
H_{R}=\sum_{p, \sigma} \epsilon_{R, p}^{0} a_{R, p \sigma}^{\dagger} a_{R, p \sigma} \\
+\sum_{p}\left[\Delta^{*} a_{R, p \downarrow} a_{R,-p \uparrow}+\Delta a_{R,-p \uparrow}^{\dagger} a_{R, p \downarrow}^{\dagger}\right],
\end{gathered}
$$

$$
\begin{gathered}
H_{\mathrm{dot}}=\sum_{i, \sigma}\left(\epsilon_{i}^{0}-e v_{g}\right) c_{i \sigma}^{\dagger} c_{i \sigma}, \\
H_{T}=\sum_{k, \sigma, i}\left[t_{L} a_{L, k \sigma}^{\dagger} c_{i \sigma}+t_{L}^{*} c_{i \sigma}^{\dagger} a_{L, k \sigma}\right] \\
+\sum_{p, \sigma, i}\left[t_{R} e^{i e v_{R} \tau} a_{R, p \sigma}^{\dagger} c_{i \sigma}+t_{R}^{*} e^{-i e v_{R} \tau^{\tau}} c_{i \sigma}^{\dagger} a_{R, p \sigma}\right],
\end{gathered}
$$

where $H_{L}$ describes the noninteracting electrons in the left normal-metal lead, $a_{L, k \sigma}^{\dagger}\left(a_{L, k \sigma}\right)$ are the creation (annihilation) operators of the electron in the left lead, and $v_{L}$ is the voltage of the left lead. $H_{R}$ describes the right superconducting lead with the energy gap $\Delta . H_{\text {dot }}$ is the Hamiltonian of the quantum dot with multiple discrete energy levels, characterized by the index $i$ and spin $\sigma$. For simplicity, the intradot electron-electron Coulomb interaction has been neglected. $v_{g}$ is the gate voltage which controls the energy levels in the dot. $H_{T}$ denotes the tunneling part of the Hamiltonian, and $t_{v}(v=L, R)$ is the hopping matrix and is assumed to be independent of the state $k$ of the leads and the dot state $(i, \sigma)$ for simplicity. In order to obtain the Hamiltonian (1) and (2), we have performed a unitary transformation similar to Ref. 9, so that the voltage of the right lead $v_{R}$ appears as a phase factor in the hopping elements.

Since the current is conserved, it is enough to calculate the current from the left lead to the QD from the evolution of the total number operator of the electrons in the left lead, $N_{L}=\Sigma_{k, \sigma} a_{L, k \sigma}^{\dagger} a_{L, k \sigma}:{ }^{26,27}$

$$
\begin{aligned}
I(\tau) & =-e\left\langle\dot{N}_{L}(\tau)\right\rangle=\frac{i e}{\hbar}\left\langle\left[N_{L}, H\right]\right\rangle \\
& =\frac{2 e}{\hbar} \operatorname{Re} \sum_{k, i, \sigma} t_{L}\left\langle\left\langle c_{i \sigma}(\tau) \mid a_{L, k \sigma}^{\dagger}(\tau)\right\rangle\right\rangle,
\end{aligned}
$$

where the Green function $\left\langle\left\langle A(\tau) \mid B\left(\tau^{\prime}\right)\right\rangle\right\rangle$ is defined as $\left\langle\left\langle A(\tau) \mid B\left(\tau^{\prime}\right)\right\rangle\right\rangle^{<} \equiv i\left\langle B\left(\tau^{\prime}\right) A(\tau)\right\rangle$. With the help of Dyson's equation, the Green's function $\left\langle\left\langle c_{i \sigma}(\tau) \mid a_{L, k \sigma}^{\dagger}\left(\tau^{\prime}\right)\right\rangle\right\rangle\langle$ can be written as

$$
\begin{aligned}
& \left\langle\left\langle c_{i \sigma}(\tau) \mid a_{L, k \sigma}^{\dagger}\left(\tau^{\prime}\right)\right\rangle\right\rangle \\
& =\sum_{j} \int d \tau_{1} t_{L}^{*}\left[\left\langle\left\langle c_{i \sigma}(\tau) \mid c_{j \sigma}^{\dagger}\left(\tau_{1}\right)\right\rangle\right\rangle^{r} g_{L, k}^{<}\left(\tau_{1}, \tau^{\prime}\right)\right. \\
& \left.\quad+\left\langle\left\langle c_{i \sigma}(\tau) \mid c_{j \sigma}^{\dagger}\left(\tau_{1}\right)\right\rangle\right\rangle{ }^{<} g_{L, k}^{a}\left(\tau_{1}, \tau^{\prime}\right)\right],
\end{aligned}
$$

here $\left\langle\left\langle A(\tau) \mid B\left(\tau_{1}\right)\right\rangle\right\rangle^{\tau} \equiv-i \theta\left(\tau-\tau_{1}\right)\left\langle\left\{A(\tau), B\left(\tau_{1}\right)\right\}\right\rangle$, and $g_{L, k}^{a,<}\left(\tau, \tau^{\prime}\right)$ is the exact Green's function of the electron in the left lead (normal-metal) without the coupling between the dot and the lead:

$$
\begin{gathered}
g_{L, k}^{r, a}\left(\tau, \tau^{\prime}\right)=\mp i \theta\left( \pm \tau \mp \tau^{\prime}\right) e^{-i\left(\epsilon_{L k}^{0}-e v_{L}\right)\left(\tau-\tau^{\prime}\right)}, \\
g_{L, k}^{<}\left(\tau, \tau^{\prime}\right)=i f_{L}\left(\epsilon_{L k}^{0}\right) e^{-i\left(\epsilon_{L k}^{0}-e v_{L}\right)\left(\tau-\tau^{\prime}\right)},
\end{gathered}
$$

where $f_{L}(x)=\left[\exp \left(x / k_{B} \mathcal{T}\right)+1\right]^{-1}$ is the Fermi distribution function with $\mathcal{T}$ being the temperature. Substituting the expression of $\left.\left\langle\left\langle c_{i \sigma}(\tau) \mid a_{L, k \sigma}^{\dagger}\left(\tau^{\prime}\right)\right\rangle\right\rangle\right\rangle^{<}$into Eq. (3), the sum over $k$ can be changed into an integral with the help of the density of states in the left lead, $\int d \omega \rho_{L}(\omega)$, and introducing the linewidth function $\Gamma_{L}(\omega)=2 \pi\left|t_{L}\right|^{2} \rho_{L}(\omega)$, we then have 


$$
\begin{aligned}
I(\tau) & =-\frac{4 e}{\hbar} \operatorname{Im} \int \frac{d \omega}{2 \pi} \Gamma_{L} \int_{-\infty}^{\tau} d \tau^{\prime} e^{i\left(\omega-e v_{L}\right)\left(\tau-\tau^{\prime}\right)} \sum_{i, j}\left[\left\langle\left\langle c_{i \uparrow}(\tau) \mid c_{j \uparrow}^{\dagger}\left(\tau^{\prime}\right)\right\rangle\right\rangle^{r} f_{L}(\omega)+\left\langle\left\langle c_{i \uparrow}(\tau) \mid c_{j \uparrow}^{\dagger}\left(\tau^{\prime}\right)\right\rangle\right\rangle^{<}\right] \\
& \equiv-\frac{4 e}{\hbar} \operatorname{Im} \int \frac{d \omega}{2 \pi} \Gamma_{L} \int_{-\infty}^{\tau} d \tau^{\prime} e^{i\left(\omega-e v_{L}\right)\left(\tau-\tau^{\prime}\right)}\left[\mathbf{G}^{r}\left(\tau, \tau^{\prime}\right) f_{L}(\omega)+\mathbf{G}^{<}\left(\tau, \tau^{\prime}\right)\right]_{11},
\end{aligned}
$$

where we have introduced the $2 \times 2$ matrix Green functions $\mathbf{G}^{r}\left(\tau, \tau^{\prime}\right)$ and $\mathbf{G}^{<}\left(\tau, \tau^{\prime}\right):^{9,28}$

$$
\begin{aligned}
& \mathbf{G}^{r, a}\left(\tau, \tau^{\prime}\right) \\
& \equiv \mp i \theta\left( \pm r \mp r^{\prime}\right) \\
& \times \sum_{i, j}\left(\begin{array}{ll}
\left\langle\left\{c_{i \uparrow}(\tau), c_{j \uparrow}^{\dagger}\left(\tau^{\prime}\right)\right\}\right\rangle & \left\langle\left\{c_{i \uparrow}(\tau), c_{j \downarrow}\left(\tau^{\prime}\right)\right\}\right\rangle \\
\left\langle\left\{c_{i \downarrow}^{\dagger}(\tau), c_{j \uparrow}^{\dagger}\left(\tau^{\prime}\right)\right\}\right\rangle & \left\langle\left\{c_{i \downarrow}^{\dagger}(\tau), c_{j \downarrow}\left(\tau^{\prime}\right)\right\}\right\rangle
\end{array}\right), \\
& \mathbf{G}^{<}\left(\tau, \tau^{\prime}\right)=i \sum_{i, j}\left(\begin{array}{ll}
\left\langle c_{j \uparrow}^{\dagger}\left(\tau^{\prime}\right) c_{i \uparrow}(\tau)\right\rangle & \left\langle c_{j \downarrow}\left(\tau^{\prime}\right) c_{i \uparrow}(\tau)\right\rangle \\
\left\langle c_{j \uparrow}^{\dagger}\left(\tau^{\prime}\right) c_{i \downarrow}^{\dagger}(\tau)\right\rangle & \left\langle c_{j \downarrow}\left(\tau^{\prime}\right) c_{i \downarrow}^{\dagger}(\tau)\right\rangle
\end{array}\right) .
\end{aligned}
$$

In order to obtain the expression of the current $I$, we have to solve the Green's functions $G_{11}^{r}\left(\tau, \tau^{\prime}\right)$ and $G_{11}^{<}\left(\tau, \tau^{\prime}\right)$. First, we solve $G_{11}^{r}\left(\tau, \tau^{\prime}\right)$ and $G_{12}^{r}\left(\tau, \tau^{\prime}\right)$ by using the Dyson equation:

$$
\begin{aligned}
\mathbf{G}^{r}\left(\tau, \tau^{\prime}\right)= & \mathbf{g}^{r}\left(\tau, \tau^{\prime}\right)+\iint d \tau_{1} d \tau_{2} \mathbf{G}^{r}\left(\tau, \tau_{1}\right) \\
& \times \Sigma^{r}\left(\tau_{1}, \tau_{2}\right) \mathbf{g}^{r}\left(\tau_{2}, \tau^{\prime}\right),
\end{aligned}
$$

where $\Sigma^{r}\left(\tau_{1}, \tau_{2}\right)$ is the self-energy function, and the Green's function $\mathbf{g}^{r}\left(\tau, \tau^{\prime}\right)$ is given by

$$
\begin{aligned}
\mathbf{g}^{r}\left(\tau, \tau^{\prime}\right) & \\
= & -i \theta\left(\tau-\tau^{\prime}\right) \\
& \times\left(\begin{array}{ll}
\sum_{i} e^{-i\left(\epsilon_{i}^{0}-e v_{g}\right)\left(\tau-\tau^{\prime}\right)} & 0 \\
0 & \sum_{i} e^{i\left(\epsilon_{i}^{0}-e v_{g}\right)\left(\tau-\tau^{\prime}\right)}
\end{array}\right) .
\end{aligned}
$$

Under the wide-bandwidth approximation, i.e., the linewidth $\Gamma_{L}(\omega)\left[\right.$ or $\left.\Gamma_{R}(\omega)\right]$ is a constant independent of $\omega,{ }^{26,27,29}$ and since the current $I$ and the probability of the Andreev reflection $T^{A}(\omega)$ depend only on $\left|t_{v}\right|^{2}$, for simplicity we assume $t_{v}$ $(v=L, R)$ is real. Then the self-energy $\Sigma^{r}\left(\tau_{1}, \tau_{2}\right)$ can be easily obtained as

$$
\Sigma^{r}\left(\tau_{1}, \tau_{2}\right)=\Sigma_{L}^{r}\left(\tau_{1}, \tau_{2}\right)+\Sigma_{R}^{r}\left(\tau_{1}, \tau_{2}\right)
$$

and

$\Sigma_{L}^{r}\left(\tau_{1}, \tau_{2}\right)=\sum_{k} t_{L} t_{L}^{*} \mathbf{g}_{L, k}^{r}\left(\tau_{1}, \tau_{2}\right)=-\frac{i \Gamma}{2} \delta\left(\tau_{1}-\tau_{2}\right)\left(\begin{array}{ll}1 & 0 \\ 0 & 1\end{array}\right)$,

$$
\begin{aligned}
\Sigma_{R}^{r}\left(\tau_{1}, \tau_{2}\right)= & \sum_{p}\left(\begin{array}{ll}
t_{R}\left(\tau_{1}\right) & 0 \\
0 & -t_{R}^{*}\left(\tau_{1}\right)
\end{array}\right) \\
& \times \mathbf{g}_{R, p}^{r}\left(\tau_{1}, \tau_{2}\right)\left(\begin{array}{ll}
t_{R}^{*}\left(\tau_{2}\right) & 0 \\
0 & -t_{R}\left(\tau_{2}\right)
\end{array}\right) \\
= & -i \Gamma_{R} \int \frac{d \omega}{2 \pi} \frac{\theta\left(\tau_{1}-\tau_{2}\right) e^{-i \omega\left(\tau_{1}-\tau_{2}\right)}}{\sqrt{\omega^{2}-\Delta^{2}}} \\
& \times\left(\begin{array}{cc}
|\omega| e^{i e v_{R}\left(\tau_{1}-\tau_{2}\right)} & -\Delta e^{i e v_{R}\left(\tau_{1}+\tau_{2}\right)} \\
-\Delta e^{-i e v_{R}\left(\tau_{1}+\tau_{2}\right)} & \left.|\omega| e^{-i e v_{R}\left(\tau_{1}-\tau_{2}\right)}\right)
\end{array}\right),
\end{aligned}
$$

where $t_{R}(\tau)$ in Eq. (14) is $t_{R} \exp \left[i e v_{R} \tau\right]$, and $\Gamma_{R}$ is defined as $2 \pi\left|t_{R}\right|^{2} \rho_{R}^{N}(\omega)$ where $\rho_{R}^{N}(\omega)$ is the density of states of the right lead in normal state. Here $\mathbf{g}_{L, k}^{r}$ and $\mathbf{g}_{R, p}^{r}$ are defined similar to Eq. (8). Notice that $\Sigma_{R}^{r}\left(\tau_{1}, \tau_{2}\right)$ has nondiagonal elements, representing the character of superconducting electrode. From Dyson's equation, Eq. (10), we have

$$
\begin{aligned}
G_{11}^{r}\left(\tau, \tau^{\prime}\right)= & g_{11}^{r}\left(\tau, \tau^{\prime}\right) \\
& +\iint d \tau_{1} d \tau_{2} G_{11}^{r}\left(\tau, \tau_{1}\right) \Sigma_{11}^{r}\left(\tau_{1}, \tau_{2}\right) g_{11}^{r}\left(\tau_{2}, \tau^{\prime}\right) \\
& +\iint d \tau_{1} d \tau_{2} G_{12}^{r}\left(\tau, \tau_{1}\right) \Sigma_{21}^{r}\left(\tau_{1}, \tau_{2}\right) g_{11}^{r}\left(\tau_{2}, \tau^{\prime}\right),
\end{aligned}
$$

$$
\begin{aligned}
G_{12}^{r}\left(\tau, \tau^{\prime}\right)= & \iint d \tau_{1} d \tau_{2} G_{11}^{r}\left(\tau, \tau_{1}\right) \Sigma_{12}^{r}\left(\tau_{1}, \tau_{2}\right) g_{22}^{r}\left(\tau_{2}, \tau^{\prime}\right) \\
& +\iint d \tau_{1} d \tau_{2} G_{12}^{r}\left(\tau, \tau_{1}\right) \Sigma_{22}^{r}\left(\tau_{1}, \tau_{2}\right) g_{22}^{r}\left(\tau_{2}, \tau^{\prime}\right) .
\end{aligned}
$$

By iterating $G_{12}^{r}\left(\tau, \tau^{\prime}\right)$ in Eqs. (16) and (15), one easily finds

$$
\begin{aligned}
G_{11}^{r}\left(\tau, \tau^{\prime}\right)= & g_{11}^{r}\left(\tau, \tau^{\prime}\right) \\
& +\iint d \tau_{1} d \tau_{2} G_{11}^{r}\left(\tau, \tau_{1}\right) \Sigma_{11}^{r}\left(\tau_{1}, \tau_{2}\right) g_{11}^{r}\left(\tau_{2}, \tau^{\prime}\right) \\
& +\iiint \int d \tau_{1} d \tau_{2} d \tau_{3} d \tau_{4} G_{11}^{r}\left(\tau, \tau_{1}\right) \\
& \times \Sigma_{12}^{r}\left(\tau_{1}, \tau_{2}\right) A^{r}\left(\tau_{2}, \tau_{3}\right) \Sigma_{21}^{r}\left(\tau_{3}, \tau_{4}\right) g_{11}^{r}\left(\tau_{4}, \tau^{\prime}\right)
\end{aligned}
$$

where we have introduced a new quantity $A^{r}\left(\tau, \tau^{\prime}\right)$ defined as 


$$
\begin{aligned}
A^{r}\left(\tau, \tau^{\prime}\right)= & g_{22}^{r}\left(\tau, \tau^{\prime}\right)+\iint d \tau_{1} d \tau_{2} g_{22}^{r}\left(\tau, \tau_{1}\right) \\
& \times \Sigma_{22}^{r}\left(\tau_{1}, \tau_{2}\right) g_{22}^{r}\left(\tau_{2}, \tau^{\prime}\right) \\
& +\iiint \int d \tau_{1} d \tau_{2} d \tau_{3} d \tau_{4} g_{22}^{r}\left(\tau, \tau_{1}\right) \Sigma_{22}^{r}\left(\tau_{1}, \tau_{2}\right) \\
& \times g_{22}^{r}\left(\tau_{2}, \tau_{3}\right) \Sigma_{22}^{r}\left(\tau_{3}, \tau_{4}\right) g_{22}^{r}\left(\tau_{4}, \tau^{\prime}\right)+\cdots
\end{aligned}
$$

which can be calculated exactly by substituting the Green's function $g_{22}^{r}$ of Eq. (11), and the self-energy function $\Sigma_{22}^{r}$ of Eq. (12), into Eq. (18),

$$
\begin{aligned}
A^{r}\left(\tau, \tau^{\prime}\right)= & \int \frac{d \omega}{2 \pi} e^{-i\left(\omega+e v_{R}\right)\left(\tau-\tau^{\prime}\right)} \\
& \times\left[\left(\sum_{i} \frac{1}{\omega+\epsilon_{i}^{0}-e v_{g}+e v_{R}}\right)^{-1}\right. \\
& \left.+\frac{i \Gamma_{L}}{2}+\frac{i \Gamma_{R}}{2} \frac{|\omega|}{\sqrt{\omega^{2}-\Delta^{2}}}\right]^{-1} \\
\equiv & \int \frac{d \omega}{2 \pi} e^{-i\left(\omega+e v_{R}\right)\left(\tau-\tau^{\prime}\right)} A^{r}(\omega) .
\end{aligned}
$$

Then substituting $A^{r}\left(\tau, \tau^{\prime}\right), \Sigma^{r}\left(\tau, \tau^{\prime}\right)$, and $\mathbf{g}^{r}\left(\tau, \tau^{\prime}\right)$ into Eq. (17), the Green's function $G_{11}^{r}\left(\tau, \tau^{\prime}\right)$ is easily obtained:

$$
G_{11}^{r}\left(\tau, \tau^{\prime}\right)=\int \frac{d \omega}{2 \pi} e^{-i\left(\omega-e v_{R}\right)\left(\tau-\tau^{\prime}\right)} \widetilde{G}_{11}^{r}(\omega),
$$

where $\widetilde{G}_{11}^{r}(\omega)$ is the Fourier transform of $G_{11}^{r}\left(\tau-\tau^{\prime}\right)$ given by

$$
\begin{aligned}
\widetilde{G}_{11}^{r}(\omega)= & {\left[\left(\sum_{i} \frac{1}{\omega-\epsilon_{i}^{0}+e v_{g}-e v_{R}}\right)^{-1}+\frac{i \Gamma_{L}}{2}\right.} \\
& \left.+\frac{i \Gamma_{R}}{2} \frac{|\omega|}{\sqrt{\omega^{2}-\Delta^{2}}}+\frac{\Gamma_{R}^{2} \Delta^{2}}{4\left(\omega^{2}-\Delta^{2}\right)} A^{r}(\omega)\right]^{-1} .
\end{aligned}
$$

Notice that the Green's function $G_{11}^{r}\left(\tau, \tau^{\prime}\right)$ is only a function of the time difference, $\tau-\tau^{\prime}$.

Then we solve the retarded Green's function $G_{12}^{r}\left(\tau, \tau^{\prime}\right)$ which is needed for solving the Green's function $G_{11}^{<}\left(\tau, \tau^{\prime}\right)$. By iterating $G_{12}^{r}\left(\tau, \tau^{\prime}\right)$ in Eq. (16), we obtain

$$
G_{12}^{r}\left(\tau, \tau^{\prime}\right)=\iint d \tau_{1} d \tau_{2} G_{11}^{r}\left(\tau, \tau_{1}\right) \Sigma_{12}^{r}\left(\tau_{1}, \tau_{2}\right) A^{r}\left(\tau_{2}, \tau^{\prime}\right)
$$

With the help of Eqs. (12)-(14), (19), and (20), the Green's function $G_{12}^{r}\left(\tau, \tau^{\prime}\right)$ is found to be

$$
G_{12}^{r}\left(\tau, \tau^{\prime}\right)=\int \frac{d \omega}{2 \pi} e^{-i \omega\left(\tau-\tau^{\prime}\right)} e^{i e v_{R}\left(\tau+\tau^{\prime}\right)} \widetilde{G}_{12}^{r}(\omega),
$$

where

$$
\widetilde{G}_{12}^{r}(\omega)=\widetilde{G}_{11}^{r}(\omega) \frac{i \Gamma_{R} \Delta}{2 \sqrt{\omega^{2}-\Delta^{2}}} A^{r}(\omega) .
$$

Notice that the Green's function $G_{12}^{r}\left(\tau, \tau^{\prime}\right)$ is not a function of the time difference except for $v_{R}=0$. Also, that $\widetilde{G}_{12}^{r}(\omega)$ in Eq. (22) is the Fourier transform of $G_{12}^{r}\left(\tau, \tau^{\prime}\right)$ in the special case with $v_{R}=0$.

In the following, we solve the Green's function $G_{11}^{<}(\tau, \tau)$ by using the Keldysh equation: $\mathbf{G}^{<}=\left(\mathbf{1}+\mathbf{G}^{r} \Sigma^{r}\right) \mathbf{g}^{<}(\mathbf{1}$ $\left.+\Sigma^{a} \mathbf{G}^{a}\right)+\mathbf{G}^{r} \Sigma^{<} \mathbf{g}^{a}$. One can easily find that the first term on the right-hand side is zero. ${ }^{26}$ Under the wide-bandwidth approximation, the self-energy $\Sigma^{<}\left(\tau_{1}, \tau_{2}\right)$ can be obtained as

$$
\Sigma^{<}\left(\tau_{1}, \tau_{2}\right)=\Sigma_{L}^{<}\left(\tau_{1}, \tau_{2}\right)+\Sigma_{R}^{<}\left(\tau_{1}, \tau_{2}\right)
$$

with

$$
\begin{aligned}
\Sigma_{L}^{<}\left(\tau_{1}, \tau_{2}\right)= & \sum_{k} t_{L} t_{L}^{*} \mathbf{g}_{L, k}^{<}\left(\tau_{1}, \tau_{2}\right) \\
= & i \Gamma_{L} \int \frac{d \omega}{2 \pi} e^{-i \omega\left(\tau_{1}-\tau_{2}\right)} \\
& \times\left(\begin{array}{ll}
f_{L}\left(\omega+e v_{L}\right) & 0 \\
0 & f_{L}\left(\omega-e v_{L}\right)
\end{array}\right)
\end{aligned}
$$

and

$$
\begin{aligned}
& \Sigma_{R}^{<}\left(\tau_{1}, \tau_{2}\right) \\
& =\sum_{p}\left(\begin{array}{ll}
t_{R}\left(\tau_{1}\right) & 0 \\
0 & -t_{R}^{*}\left(\tau_{1}\right)
\end{array}\right) \\
& \times g_{R, p}^{<}\left(\tau_{1}, \tau_{2}\right)\left(\begin{array}{ll}
t_{R}^{*}\left(\tau_{2}\right) & 0 \\
0 & -t_{R}\left(\tau_{2}\right)
\end{array}\right) \\
& =i \Gamma_{R} \int \frac{d \omega}{2 \pi} e^{-i \omega\left(\tau_{1}-\tau_{2}\right)} f_{R}(\omega) \widetilde{\rho}_{R}(\omega) \\
& \times\left(\begin{array}{ll}
e^{i e v_{R}\left(\tau_{1}-\tau_{2}\right)} & -\frac{\Delta}{|\omega|} e^{i e v_{R}\left(\tau_{1}+\tau_{2}\right)} \\
-\frac{\Delta}{|\omega|} e^{-i e v_{R}\left(\tau_{1}+\tau_{2}\right)} & e^{-i e v_{R}\left(\tau_{1}-\tau_{2}\right)}
\end{array}\right),
\end{aligned}
$$

where $f_{R}(\omega)=f_{L}(\omega)$ is the Fermi distribution function, and $\widetilde{\rho}_{R}(\omega)$ is the corresponding dimensionless BCS density of states, i.e., $\widetilde{\rho}_{R}(\omega)$ is the ratio of superconducting density of states, $\rho_{R}^{S}(\omega)$, to the normal density of states, $\rho_{R}^{N}(\omega): \widetilde{\rho}_{R}(\omega) \equiv \rho_{R}^{S}(\omega) / \rho_{R}^{N}(\omega)$. From

$$
\rho_{R}^{S}(\omega)=(1 / \pi) \operatorname{Im} \sum_{p} g_{R, p}^{a}(\omega),
$$

one easily finds 9,20

$$
\widetilde{\rho}_{R}(\omega)=\theta(|\omega|-\Delta)|\omega| / \sqrt{\omega^{2}-\Delta^{2}} .
$$

Substituting the expressions of the self-energy $\Sigma<\left(\tau_{1}, \tau_{2}\right)$, Eqs. (23)-(25), and the Green's functions $G_{11}^{r}\left(\tau, \tau^{\prime}\right)$, and $G_{12}^{r}\left(\tau, \tau^{\prime}\right)$ into the Keldysh equation, then $\mathbf{G}_{11}^{<}(\tau, \tau)$ can be obtained straightforwardly, 


$$
\begin{aligned}
\mathbf{G}_{11}^{<}(\tau, \tau)= & \iint d \tau_{1} d \tau_{2}\left[\mathbf{G}_{11}^{r} \Sigma_{11}^{<} \mathbf{G}_{11}^{a}+\mathbf{G}_{11}^{r} \Sigma_{12}^{<} \mathbf{G}_{21}^{a}+\mathbf{G}_{12}^{r} \Sigma_{21}^{<} \mathbf{G}_{11}^{a}+\mathbf{G}_{12}^{r} \Sigma_{22}^{<} \mathbf{G}_{21}^{a}\right] \\
= & \int \frac{d \omega}{2 \pi}\left|\tilde{G}_{11}^{r}(\omega)\right|^{2} i \Gamma_{L} f_{L}\left(\omega+e v_{L}-e v_{R}\right)+\int \frac{d \omega}{2 \pi}\left|\tilde{G}_{12}^{r}(\omega)\right|^{2} i \Gamma_{L} f_{L}\left(\omega-e v_{L}+e v_{R}\right) \\
& +\int \frac{d \omega}{2 \pi} i \Gamma_{R} \widetilde{\rho}_{R}(\omega) f_{R}(\omega)\left\{\left|\widetilde{G}_{11}^{r}(\omega)\right|^{2}+\left|\widetilde{G}_{12}^{r}(\omega)\right|^{2}-\frac{2 \Delta}{|\omega|} \operatorname{Re}\left[\widetilde{G}_{11}^{r}(\omega)\left(\widetilde{G}_{12}^{r}(\omega)\right)^{*}\right]\right\} .
\end{aligned}
$$

Notice that for the N-QD-S system, the current should be independent of time for the dc bias. As expected, the Green's function $G_{11}^{<}(\tau, \tau)$ does not depend on time $\tau$, although $G_{12}^{r}\left(\tau, \tau^{\prime}\right)$ and $\Sigma_{12}^{r}\left(\tau, \tau^{\prime}\right)$ depend on two time variables $\tau$ and $\tau^{\prime}$.

Finally, substituting the retarded Green's function $G_{11}^{r}\left(\tau, \tau^{\prime}\right)$, Eq. (20), and the distribution Green's function $G_{11}^{<}(\tau, \tau)$, Eq. (27), into the expression of the current, Eq. (7), then the current is obtained as

$$
I=I_{A}+I_{1},
$$

with

$$
\begin{aligned}
I_{A}= & \frac{2 e \Gamma_{L}^{2}}{\hbar} \int \frac{d \omega}{2 \pi}\left|\widetilde{G}_{12}^{r}\right|^{2}\left[f_{L}\left(\omega+e v_{L}-e v_{R}\right)\right. \\
& \left.-f_{L}\left(\omega-e v_{L}+e v_{R}\right)\right], \\
I_{1}= & \frac{2 e \Gamma_{L} \Gamma_{R}}{\hbar} \int \frac{d \omega}{2 \pi} \widetilde{\rho}_{R}(\omega)\left[\left|\widetilde{G}_{11}^{r}\right|^{2}+\left|\widetilde{G}_{12}^{r}\right|^{2}\right. \\
& \left.-\frac{2 \Delta}{|\omega|} \operatorname{Re}\left[\widetilde{G}_{11}^{r}(\omega)\left(\widetilde{G}_{12}^{r}(\omega)\right)^{*}\right]\right] \\
& \times\left[f_{L}\left(\omega+e v_{L}-e v_{R}\right)-f_{R}(\omega)\right] .
\end{aligned}
$$

The formulas of the current, Eqs. (28) and (29), are the central results of this paper. Obviously, the expression of the current for the N-QD-S system under consideration is independent on the time $\tau$, and only depends on the dc bias voltage $V$. If the voltages of the left lead, the right lead, and the gate $\left(v_{L}, v_{R}\right.$, and $\left.v_{g}\right)$ are shifted by the same amount $\Delta V$, the current $I_{1}$ and $I_{A}$ do not change, i.e., they satisfy the gauge-invariant condition. ${ }^{30}$ The current $I_{A}$ represents the contribution from the Andreev reflection, in which an electron incoming from the left lead will be reflected as a hole backwards into the left lead and an extra Cooper pair is created in the right superconducting lead. The current $I_{1}$ consists of three different processes: ${ }^{9}$ (1) The conventional electron tunneling through the system, i.e., the term $\Gamma_{L} \Gamma_{R} \widetilde{\rho}_{R}\left|\widetilde{G}_{11}^{r}(\omega)\right|^{2}$; (2) An electron incident from the left lead will convert into a hole like in the right superconducting lead, i.e., the term $\Gamma_{L} \Gamma_{R} \widetilde{\rho}_{R}\left|\widetilde{G}_{12}^{r}(\omega)\right|^{2}$, corresponding to a "branch crossing" process in Blonder-Tinkham-Klapwijk (BTK) theory; ${ }^{25}$ (3) An electron (or a hole) incident from the left lead tunnels into the right superconducting lead, picks up a quasiparticle (or a quasihole) in the superconductor and creates (or annihilates) a Cooper pair, i.e., the term $-\Gamma_{L} \Gamma_{R} \widetilde{\rho}_{R}(2 \Delta /|\omega|) \operatorname{Re}\left[\widetilde{G}_{11}^{r} \widetilde{G}_{12}^{r *}\right]$. It should be pointed out that at zero temperature if $|e V|<\Delta$ one has $I_{1}=0$, i.e., only the Andreev reflection process contributes to the current. However, when $|e V|>\Delta$, all of the processes mentioned above make the contributions to the current.

Finally, the probability of the Andreev reflection $T^{A}(\omega)$ can be easily found from the expression of the current $I_{A}$ due to the Andreev reflection, Eq. (28), as

$$
T^{A}(\omega)=\Gamma_{L}^{2}\left|\widetilde{G}_{12}^{r}(\omega)\right|^{2} .
$$

\section{DIFFERENT SERIES OF RESONANT ANDREEV REFLECTION PEAKS}

On the basis of the current formulas, Eqs. (28), and (29), we investigate the properties of the current $I$ versus the gate voltage $v_{g}$ (or the gate-induced charge $Q$ ). Unlike the system of N-QD-N in which the current $I$ versus the gate voltage $v_{g}$ at small bias $V$ exhibits a single series of equally spaced resonance peaks. Now with one of the leads replaced by a superconducting lead, the situation becomes more complicated. In the following we shall discuss in detail two different cases for $e V<\Delta$ and $e V>\Delta$. We set (1) the temperature $\mathcal{T}=0$, (2) the voltage of the right leads $v_{R}=0$ due to the gauge invariance, and carry out all calculations in units of $\hbar=e=1$.

\section{A. The case with $V<\Delta$}

In the case of $V<\Delta$, only the Andreev reflection current $I_{A}$ exists, i.e., $I_{1}=0$. Figure 1 presents the current $I$ vs the gate voltage $v_{g}$ at different bias. In numerical studies, we have assumed that the QD has ten discrete levels (from $\epsilon_{0}$ to $\left.\epsilon_{9}\right)$ with equal level spacing. These levels move downward as $v_{g}$ increases. At small bias, the current $I$ vs the gate voltage $v_{g}$ exhibits a single series of peaks with equal spacing [marked with " $A$ " in Fig. 1(a)]. We emphasize that although these resonance peaks are similar to those peaks in a normal $\mathrm{N}-\mathrm{QD}-\mathrm{N}$ system, they are not due to from the conventional resonant tunneling, because the conventional tunneling is completely forbidden for $V<\Delta$. In fact, these peaks come from the Andreev reflections. When the energy of a state $i$ of the QD is lined up with the chemical potential of the right superconducting lead, $\mu_{R}\left(\mu_{R}=-v_{R}\right.$ is set to be zero), then an electron incident from the left lead with the energy $\epsilon$ $=\mu_{R}$ can tunnel into the state $i$ of the QD, leading to a hole propagating back to the state $i$ in the QD and the creation of a Cooper pair in the right superconducting lead because of Andreev reflection [see Fig. 2(a)]. As a result, a current peak emerges in the $I-v_{g}$ curve. Notice that in this Andreev re- 


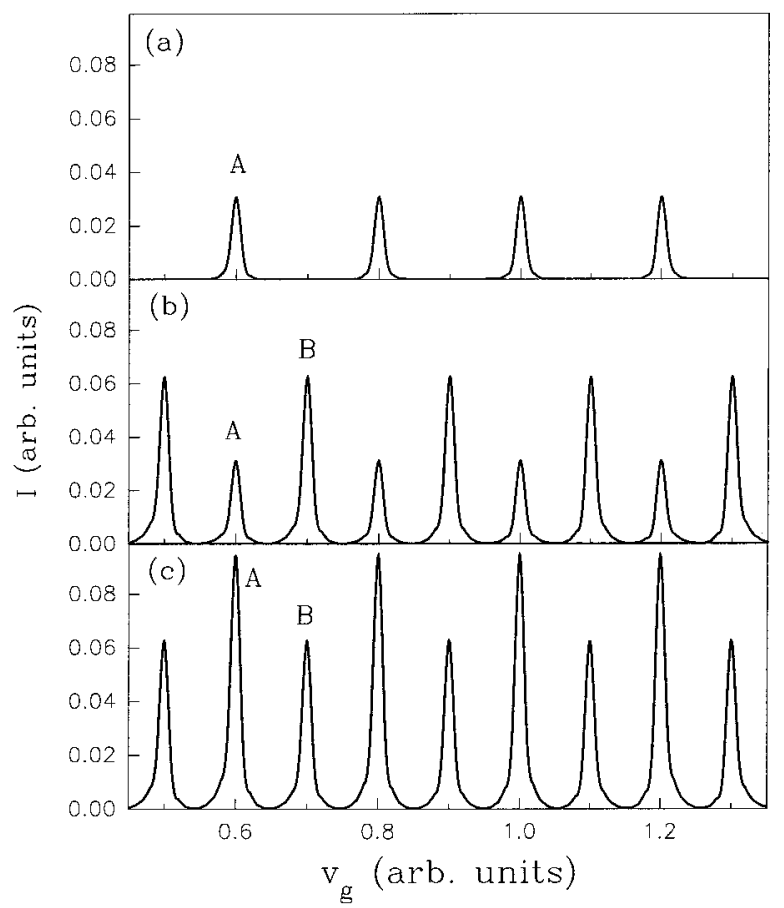

FIG. 1. The current $I$ vs the gate voltage $v_{g}$ for $V<\Delta$. The QD has ten states with equal level spacing, $\Delta \epsilon=0.2$; and $\epsilon_{0}=0$ at $v_{g}$ $=0$. Other parameters are chosen as $\Gamma_{L}=\Gamma_{R}=0.01, \Delta=1$. (a), (b), and (c) correspond to three different bias: $V=0.02,0.15$, and 0.25 , respectively.

flection process the incident electron and the reflected hole go through the same state $i$ of the QD. If the energy level is not lined up with $\mu_{R}$, the Andreev reflection will be very weak, corresponding to a very small Andreev reflection current $I_{A}$.

Gradually increasing the bias $V$, the basic feature of the current $I$ versus the gate voltage $v_{g}$ does not change much, as long as $V<\Delta \epsilon / 2$, where $\Delta \epsilon$ is the level spacing of the QD. Hence the corresponding differential conductance $d I / d V$ is almost zero for $V<\Delta \epsilon / 2$. However, when $V$ is greater than $\Delta \epsilon / 2$, a series of extra peaks emerges in the $I-v_{g}$ curve [marked with " $B$ "' in Fig. 1(b)]. The $B$-type peaks are located in the middle of the $A$-type peaks with the same peak spacing as that of the $A$-type peaks, but have much larger amplitudes than that of the $A$-type peaks (approximately doubled).

The $B$-type peaks are originated from the following Andreev reflection. For a certain gate voltage $v_{g}$, two neighboring states of the QD, say $\epsilon_{i}$ and $\epsilon_{i+1}$, satisfy two condi-

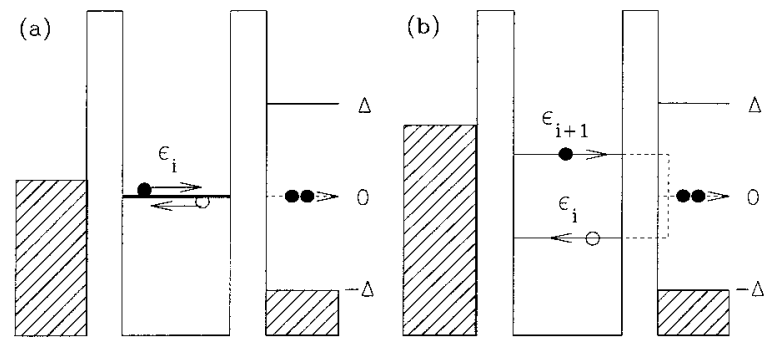

FIG. 2. A schematic diagram for the Andreev reflections: (a) the Andreev reflection through the state $i$ itself; (b) the Andreev reflection through two states, the state $i$ and state $(i+1)$. tions: the chemical potential of the right superconducting lead $\mu_{R}$, is located in the middle of the two levels; while both of these two levels are below the chemical potential of the left lead, $\mu_{L}\left(\mu_{L}=-v_{L}\right)$. So an electron incident with energy $\epsilon=\epsilon_{i+1}$ (or $\epsilon_{i}$ ) tunnels from the left lead through the left barrier into the QD's state $(i+1)$ (or state $i$ ), then Andreev reflected at the right barrier as a hole back to the QD's state $i$ [or state $(i+1)]$, with a Cooper pair created in the superconductor in the meantime [see Fig. 2(b)]. Notice that in the $B$-type Andreev reflection both state $(i+1)$ and state $i$ contribute to the current, while the $A$-type Andreev reflection only involves a single state, so the $B$-type peak has approximately twice the amplitude as that of the $A$-type peak. It should be emphasized that if $V<\Delta \epsilon / 2$, the $B$-type Andreev reflection cannot happen at zero temperature for the following reasons: (1) The electron cannot tunnel from the left lead to the QD's state $(i+1)$ with $\mu_{L}<\epsilon_{i+1}$ since the state $(i+1)$ is empty, in another words, it is occupied by a hole. (2) Although an electron can tunnel from the left lead to the QD's state $i$, the Andreev reflection is prohibited since the state $(i+1)$ has already been occupied by a hole.

When $V$ is less than $\Delta \epsilon$ the amplitude of the $A$-type peak is smaller than that of the $B$-type peak. As the bias $V$ is larger than $\Delta \epsilon$ (within the energy range of $\Gamma$ ), the amplitude of the $A$-type peak becomes larger than that of $B$-type peak and eventually reaches a height three times it original value [see Fig. 1(c)]. The positions of the $A$-type peaks do not change. In fact, for a certain gate voltage $v_{g}$, a QD's state $i$ will line up with the chemical potential $\mu_{R}$, so the Andreev reflection through the state $i$ itself replaces the original $A$-type peak. Meanwhile, not only the state $(i+1)$ and the state $(i-1)$ are just at $\Delta \epsilon$ and $-\Delta \epsilon$, respectively, both of them are below the left chemical potential $\mu_{L}$ as well, due to the condition $V$ $>\Delta \epsilon$. So the Andreev reflection can also occur through the state $(i+1)$ and state $(i-1)$. Now three states $(i-1), i$, and $(i+1)$ contribute to the $A$-type peaks, resulting in a much higher peak.

It should be mentioned that in the above numerical calculation, we have assumed that the energy levels in the dot are equally spaced. If the level spacings are unequal, the results will be more complicated. Generally, the interval between peaks of each type becomes different from peak to peak. To see this change concretely, let us consider a special case of the quantum dot with ten energy levels and the level spacing as $\Delta \epsilon_{i}=0.17$ for even $i$, and $\Delta \epsilon_{i}=0.23$ for odd $i\left(\Delta \epsilon_{i}\right.$ $\left.\equiv \epsilon_{i+1}-\epsilon_{i}\right)$. First, at the small bias case, i.e., $V<\Delta \epsilon_{i} / 2$, the $I-v_{g}$ curve only has a single series of peaks (type- $A$ ) with the same height as in Fig. 1(a), but the intervals are not equal (not shown here). Second, with the increase of the bias $V$, from smaller than $\Delta \epsilon_{i} / 2$ to larger than $\Delta \epsilon_{i} / 2$, a different $B$-type peak will emerge at different bias $V$ due to the dependence of $\Delta \epsilon_{i} / 2$ on $i$. The peaks corresponding to smaller $\Delta \epsilon_{i}$ will emerge first, i.e., at a smaller value of $V$; and the peaks corresponding to larger $\Delta \epsilon_{i}$ will emerge at larger $V$. Notice that there is no change of the heights of all peaks, and the locations of the $B$-type peaks are still in the middle between the $A$-type peaks (not shown here). Figure 3 shows the case of $V>\Delta \epsilon_{i}$. In contrast to the equal-spacing case [compare to Fig. 1(c)], the original $A$-type peaks are splitted into two peaks (marked with $A_{1}$ and $A_{2}$ in Fig. 3). The reason is: The $A$-type peaks in Fig. 1(c) originate from two kinds of 


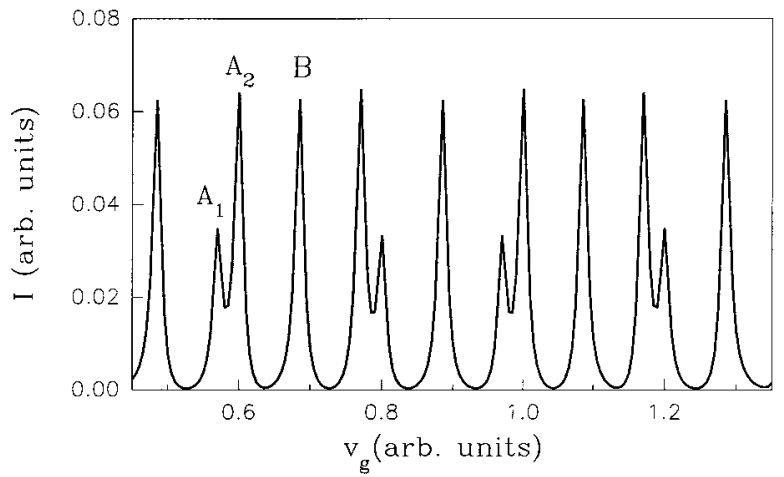

FIG. 3. The current $I$ vs the gate voltage $v_{g}$ for $V<\Delta$. The QD has ten states but with unequal level spacing: $\Delta \epsilon_{i}=0.17$ for even $i$, $\Delta \epsilon_{i}=0.23$ for odd $i$, and $\epsilon_{0}=0$ at $v_{g}=0$. The other parameters are the same as in Fig. 1(c).

Andreev reflections, one is the Andreev reflection through the state $i$ itself, another through the state $(i+1)$ and state $(i-1)$. In the equal-spacing case, these two completely overlap to combine into a much higher $A$-type peak. However, for the unequal level-spacing case, the two peaks from the two kinds of Andreev reflections described above no longer overlap completely. So one sees a series of splitted two-peak resonances. In the rest of this paper, we will keep the assumption of equal level spacing for simplicity.

\section{B. The case with $V>\Delta$}

In the case of $V>\Delta$, all kinds of the processes contribute to the current $I$, including Andreev reflection and all three kinds of tunneling processes mentioned above, i.e., both $I_{A}$ and $I_{1}$ are nonzero.

Here we studied the case of $V$ slightly larger than the energy gap $\Delta$. Figure 4 shows the current $I$ vs the gate voltage $v_{g}$ in this case. The difference between Figs. 4(a) and 4(b) is due to the different level spacing of the QD's states. Now three series of peaks emerge in the curve of $I$ vs $v_{g}$ (marked by $A, B$, and $C$, respectively), and with the same intervals (or periods) between the peaks for each series. The positions of the $B$-type peaks are in the middle of the $A$-type peaks; and the position of the $C$-type peak is shifted from the $A$-type peak by a certain amount which depends on the level spacing $\Delta \epsilon$ and the gap $\Delta$. The height of the $C$-type peak is the smallest one.

To understand the origin and the position of each kind of peak, in the following numerical studies, we fix the parameters as indicated in Fig. 4(a), and set $\Delta \epsilon=0.8$. Since $\epsilon_{i}$ $=\epsilon_{i}^{0}-v_{g}$, which varies with the gate voltage $v_{g}$, the state $\epsilon_{4}$ is just below the chemical potential $\mu_{L}$ and above the gap $\Delta$ when $v_{g}=2.2$ [see Fig. 5(a)]. So the conventional electron and quasiparticle tunneling processes are allowed, leading to the $C$-type peaks. With the increase of the gate voltage, each level of the QD moves downward. When $v_{g}=2.4$, the state $\epsilon_{3}$ is lined up with the chemical potential $\mu_{R}$, and the energy difference between the state $\epsilon_{4}$ or $\epsilon_{2}$ and $\mu_{R}$ are equal [see Fig. 5(b)]. So the Andreev reflection can occur through either the state $\epsilon_{3}$ itself or the other two states $\epsilon_{4}$ and $\epsilon_{2}$, leading to the $A$-type peaks with the largest amplitude [see Fig. 4(a)]. As $v_{g}$ is increased further at $v_{g}=2.8$, the energy difference between the state $\epsilon_{4}$ or state $\epsilon_{3}$ and $\mu_{R}$ are equal (not shown
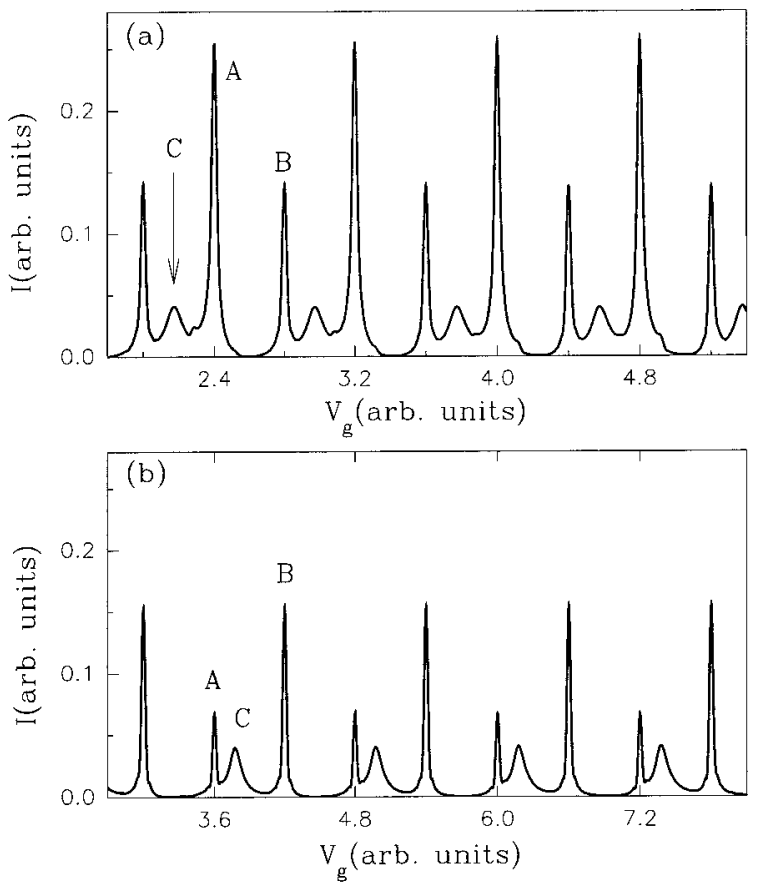

FIG. 4. The current $I$ vs the gate voltage $v_{g}$ for $V>\Delta$. The QD has ten states with equal level spacing; and $\epsilon_{0}=0$ at $v_{g}=0$. Other parameters are chosen as $\Gamma_{L}=\Gamma_{R}=0.02, \Delta=1$, and $V=1.04$. (a) and (b) correspond to $\Delta \epsilon=0.8$ and 1.2 , respectively.

here), and both $\epsilon_{3}$ and $\epsilon_{4}$ are below the chemical potential of the left lead, $\mu_{L}$. Then the Andreev reflection occurs through the states $\epsilon_{4}$ and $\epsilon_{3}$, corresponding to the $B$-type peaks. For the case with symmetric barriers (as assumed in our calculations), i.e., $\Gamma_{L}=\Gamma_{R}$, the maximum probability of the Andreev reflection can reach one (see Sec. IV below), but the conventional transmission probability is less than one because $\Gamma_{L} \neq \Gamma_{R} \widetilde{\rho}_{R}$. Therefore the height of the $C$-type peak is the smallest one.

\section{PROBABILITY OF THE ANDREEV REFLECTION}

Now we turn to investigate the probability of the Andreev reflection, $T^{A}(\omega)$. Figure 6(a) shows the dependence of $T^{A}(\omega)$ on the energy $\omega$. Here we have assumed that the QD only has two states $\left(\epsilon_{0}\right.$, and $\left.\epsilon_{1}\right)$ with $\epsilon_{0}=-0.25$ and $\epsilon_{1}$ $=0.25$, i.e., the energy difference between $\epsilon_{0}$ or $\epsilon_{1}$ and the chemical potential $\mu_{R}$ are equal. Two peaks emerge in the
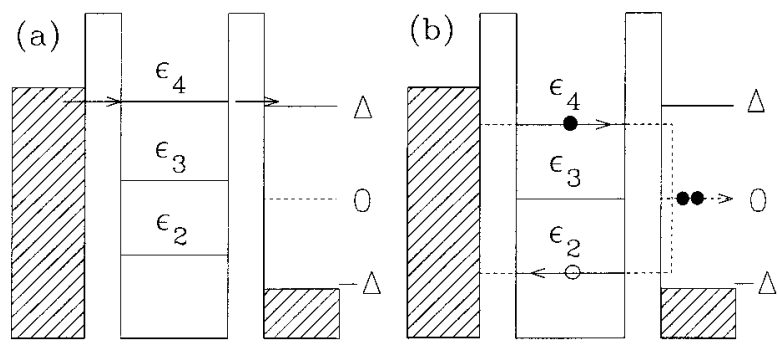

FIG. 5. A schematic diagram for the tunneling processes responsible for the $C$-type and the $A$-type peaks. (a) and (b) describe the positions of the QD's states, and show the related conventional tunneling ( $C$-type) and the Andreev tunneling (A-type) processes, respectively. 


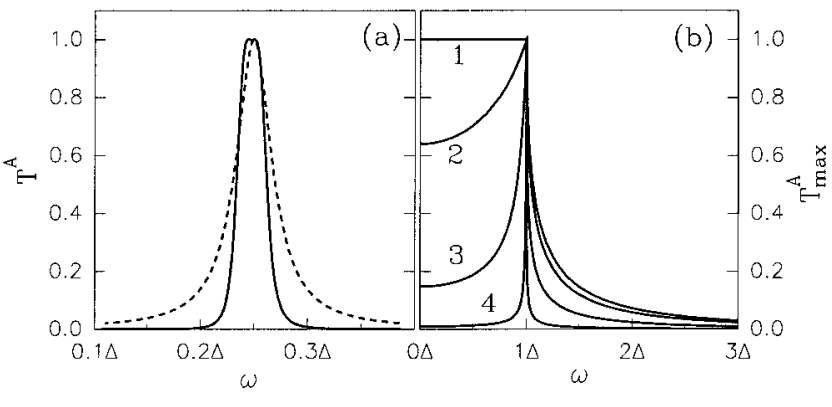

FIG. 6. (a) The probability of the Andreev reflection $T^{A}(\omega)$ vs the energy $\omega$. The QD has two states $\left(\epsilon_{0}, \epsilon_{1}\right)$ with $\epsilon_{0}=-0.25$ and $\epsilon_{1}=0.25$. Other parameters are chosen as $\Gamma_{L}=\Gamma_{R}=0.02, \Delta=1$. The dotted curve shows the Breit-Winger transmission probability $T(\omega)$ for comparison. (b) The resonant Andreev reflection probability $T_{\max }^{A}(\omega)$ vs $\omega$ at different ratios of $\Gamma_{R} / \Gamma_{L}$. The curves $1,2,3$, and 4 correspond to $\Gamma_{L} / \Gamma_{R}=1,2,5$, and 20, respectively; with $\Gamma_{L}=0.01$.

curve of $T^{A}(\omega)$ versus $\omega$, one at $\omega=\epsilon_{1}$ and the other at $\epsilon_{0}$. Only the peak at $\omega=\epsilon_{1}$ is shown in Fig. 6(a). Notice that in the case of symmetric barriers, the maximum probability of the Andreev reflection is one, and is independent of $\Gamma_{L}$ (or $\left.\Gamma_{R}\right)$. It is well known from BTK theory ${ }^{25}$ that the probability of the Andreev reflection for a single barrier is related to the transmission probability through the barrier (or the height of the barrier). The smaller the transmission probability of the barrier, the smaller the Andreev reflection probability. The probability of the Andreev reflection can reach one only if there is no barrier at all. Usually, for a realistic single-barrier $\mathrm{N}-\mathrm{S}$ structure, the probability of the Andreev reflection is very small, much smaller than one. ${ }^{5}$ However, in the system with two barriers like the one under consideration, the probability of the Andreev reflection exhibits strong resonant behavior, even if both $\Gamma_{L}$ and $\Gamma_{R}$ are very small. For a mesoscopic N-QD-S system, an electron (or a hole) maintains its phase coherence during the process of multiple reflections between the two barriers, the resonant Andreev reflection or the resonant Andreev tunneling can be achieved. Here the two barriers play a similar role as the two mirrors in a FabryPerot interferometer. This result is consistent with the one obtained in Refs. 22 and 3.

If the value of $\omega$ slightly deviates from $\epsilon_{1}\left(\right.$ or $\left.\epsilon_{0}\right)$, the probability of the Andreev reflection $T^{A}(\omega)$ decreases quickly as shown in Fig. 6(a), where the Breit-Winger transmission probability $T(\omega)$

$$
T(\omega)=\frac{\Gamma_{L} \Gamma_{R}}{\left(\omega-\epsilon_{1}\right)^{2}+\left(\Gamma_{L}+\Gamma_{R}\right)^{2} / 4}
$$

is also shown for comparison (the dotted line). One clearly sees that the probability of the Andreev reflection, $T^{A}(\omega)$, drops much faster than the Breit-Winger transmission probability $T(\omega)$.

In order to see the dependence of the maximum value of the Andreev reflection [denoted by $\left.T_{\max }^{A}(\omega)\right]$ on $\omega$, we consider any two states of the QD, say $\epsilon_{i}$ and $\epsilon_{j}$, and assume $\omega=\epsilon_{i}=-\epsilon_{j}$ which can be achieved by adjusting the gate voltage $v_{g}$. Now the resonant Andreev reflection occurs, and the maximum value of $T^{A}(\omega)$ can be obtained from Eq. (30):

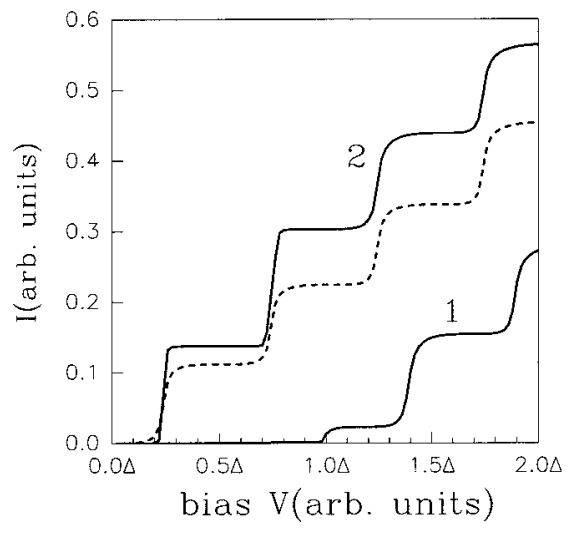

FIG. 7. The current $I$ vs the bias $V$, assuming that the QD's states are fixed relative to $\mu_{R}$. The QD has ten states (marked from $\epsilon_{0}$ to $\left.\epsilon_{9}\right)$ with $\Delta \epsilon=0.5$. Other parameters are $\Gamma_{L}=\Gamma_{R}=0.02, \Delta$ $=1$. The curves 1 and 2 correspond to $\epsilon_{5}=0.4$ and 0.25 , respectively. The dotted curve is the $I-V$ of a N-QD-N with the same parameters as the curve 2 .

$$
\begin{gathered}
T_{\max }^{A}(\omega)=\frac{4 \Gamma_{L}^{2} \Gamma_{R}^{2} \Delta^{2}}{\left(\Gamma_{L}^{2}+\Gamma_{R}^{2}\right)^{2}\left(\Delta^{2}-\omega^{2}\right)+4 \Gamma_{L}^{2} \Gamma_{R}^{2} \omega^{2}} \text { for }|\omega|<\Delta, \\
T_{\max }^{A}(\omega)=\frac{4 \Gamma_{L}^{2} \Gamma_{R}^{2} \Delta^{2}}{\left[\left(\Gamma_{L}^{2}+\Gamma_{R}^{2}\right) \sqrt{\omega^{2}-\Delta^{2}}+2 \Gamma_{L} \Gamma_{R}|\omega|\right]^{2}} \\
\text { for }|\omega|>\Delta .
\end{gathered}
$$

Figure 6(b) shows $T_{\max }^{A}(\omega)$ versus $\omega$ at different ratios of $\Gamma_{R} / \Gamma_{L}$. One can see clearly that (1) For the case of symmetric barriers $\left(\Gamma_{L}=\Gamma_{R}\right)$ and $|\omega|<\Delta$, the resonant Andreev reflection probability $T_{\max }^{A}(\omega)$ is one independent of energy $\omega$ (one has to change the gate voltage $v_{g}$ to satisfy the resonant condition). (2) If $|\omega|>\Delta, T_{\max }^{A}(\omega)$ cannot reach one even for the symmetric barriers. With the increase of $|\omega|, T_{\max }^{A}(\omega)$ drops very fast in the form of a power law $\omega^{-2}$. (3) The value of $T_{\max }^{A}(\omega)$ is always smaller for the asymmetric barriers compared with symmetric ones. (4) If $|\omega|=\Delta, T_{\max }^{A}(\omega)$ is one for both symmetric and asymmetric barriers. Notice that the dependence of $T_{\max }^{A}(\omega)$ on the energy $\omega$ at different ratio of $\Gamma_{R} / \Gamma_{L}$ is similar to the dependence of the Andreev reflection probability $A$ on the energy $\omega$ at different height $Z$ of the barrier in BTK theory. ${ }^{25}$

\section{THE I-V CHARACTERISTICS}

In this section we assume that each level of the QD remains a fixed difference with respect to $\mu_{R}$ and does not change with the bias (this can be done by varying the gate voltage simultaneously). Several observations are in order. (1) If any two intradot states do not satisfy the conditions of the resonant Andreev reflection: one is above and the other is below the chemical potential of the superconducting electrode and the differences between each state and $\mu_{R}$ are equal. Then the Andreev reflection is very small, leading to an almost invisible current $I$ for $V<\Delta$, and the plateaus in $I-V$ will not emerge until $V>\Delta$ (see curve one in Fig. 7). (2) If the conditions of the resonant Andreev reflection are satisfied, then plateaus in $I-V$ emerge even for $V<\Delta$, and the 


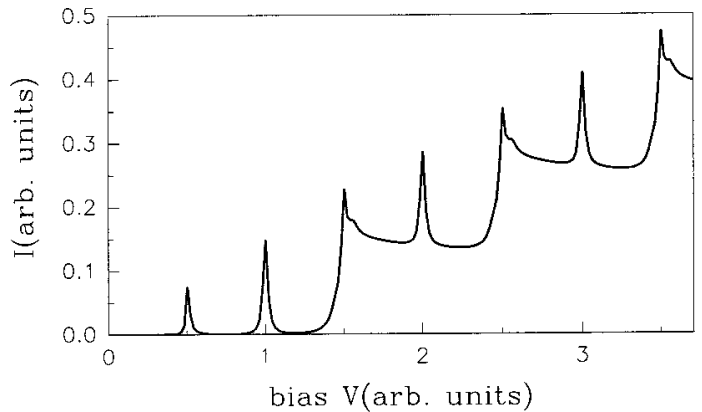

FIG. 8. The current $I$ vs bias $V$ for $\Gamma_{L}=\Gamma_{R}=0.01$ and $\Delta=1$. The QD has 20 states (marked from $\epsilon_{-10}$ to $\epsilon_{9}$ ) with $\Delta \epsilon=0.5$, and $\epsilon_{0}=0.25$ at bias $V=0$.

heights of the corresponding plateaus are higher than the ones in a N-QD-N system. The dotted curve in Fig. 7 shows the current $I$ versus the bias $V$ for a N-QD-N system for comparison, and the curve of $I$ versus $V$ is a series of approximately equal interval plateaus.

Next, we consider the case in which the levels of the QD vary with the bias $V$. In the case of symmetric barriers, we set the energies of the states as: $\epsilon_{i}=\epsilon_{i}^{0}-V / 2$. The dependence of current $I$ with the gate voltage $v_{g}$ is given in Fig. 8, where one can see extra peaks superimposed on the conventional plateaus of the $I-V$ curve. No plateau appears until $V>\Delta$; then plateaus emerge with approximate equal interval. Notice that even for $V<\Delta$ the extra peaks with equal interval due to the Andreev reflections can still emerge. This is because for a certain bias $V$, which satisfies the conditions of the resonant Andreev reflection, the current $I$ increases drastically and a peak emerges. When the bias $V$ is increased slightly, the condition of the resonant Andreev reflection is violated, and hence the current $I$ drops rapidly. The difference of the relative heights of the peaks shown in Fig. 8 is determined by the different number of states participating in the resonant Andreev reflections.

Finally, we study the $I-V$ characteristics for the case with strongly asymmetric barriers (Fig. 9). Now the BCS spectral density, $\rho_{R}^{s}(\omega)$, is directly manifested in the $I-V$ curve. This result is similar to the one obtained experimentally by Ralph, Black, and Tinkham, ${ }^{19}$ and the theoretical result by Yeyati et al. ${ }^{20}$ both of which are for a QD coupled to two superconducting leads, i.e., a S-QD-S system. Different from the system studied in Refs. 19 and 20, the system studied in this work has only one superconducting electrode.

\section{CONCLUSIONS}

In this paper, we have studied the electron tunneling through the system of a quantum dot with multiple discrete levels and coupled to two leads in which one is superconducting. The gauge-invariant formula of the current $I$ and the probability of the Andreev reflection $T^{A}(\omega)$ are derived. The resonant behavior and the resonant conditions for Andreev reflection are discussed. The resonant behavior can be clearly seen either from $I$ versus $v_{g}$ or from the dependence of the

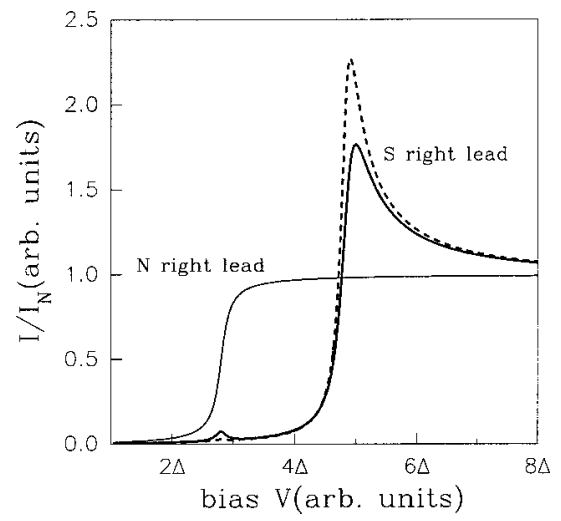

FIG. 9. The current $I$ vs the bias $V$ for strongly asymmetric barriers. Only a single state of the QD is considered, with $\epsilon_{0}=$ -1.4 at bias $V=0$. The solid and dotted curves correspond to $\Gamma_{R} / \Gamma_{L}=0.1$ and 0.02 , respectively, with $\Gamma_{L}=0.1$, and $\Delta=1$.

probability on the energy, $T^{A}(\omega)$. This means that the $\mathrm{N}$-QD-S system is quite different from the one-barrier system such as the N-S or S-S quantum point contact, in which $T^{A}(\omega)$ is always very small if the barrier is high. For the N-QD-S system with multiple levels, the $I-v_{g}$ curve has more complicated resonance patterns, with different kinds of peaks, depending on the bias $V$, the level spacing of the QD, and the energy gap of the superconducting electrode. From the study of $I-V$ curves we found some extra peaks superimposed on the conventional current plateaus, originating from Andreev reflections. In the case with highly asymmetric barriers, the $I-V$ curve can directly reflect the BCS spectral density $\widetilde{\rho}_{R}$ of the superconducting electrode, providing an alternative way to determine the BCS density of states of the quasiparticles of the superconductors.

It is worth mentioning that in this paper we have neglected the intradot Coulomb interaction. If the Coulomb interaction $U$ is included, we would expect that each kind of peak in the $I-v_{g}$ curve and the corresponding extra peaks in the $I-V$ curve will still emerge, with the same peak width. But the interval of the peaks will be widened, from $\Delta \epsilon$ to $\Delta \epsilon+U$. In addition, some substructure of the peak may emerge. The effects of the intradot Coulomb interaction on the resonant Andreev reflection will be investigated elsewhere.

\section{ACKNOWLEDGMENTS}

We gratefully acknowledge the financial support by a RGC grant from the SAR Government of Hong Kong under Grant No. HKU 7112/97P, a grant from the Croucher Foundation, a CRCG grant from the University of Hong Kong, and the research grant from the Chinese National Natural Science Foundation, and the Doctoral Program Foundation of Institution of Higher Education. We also thank the Computer Center of the University of Hong Kong for computational facilities. Q.E.S. and T.S.L. would like to thank B. Y. Gu, B. G. Wang, Q. R. Zheng, X. A. Zhao, and Y. D. Wei for many helpful discussions and for helping us with the numerical simulations. 
*Author to whom correspondence should be addressed. Permanent address: Department of Physics, Peking University, Beijing 100871, China.

${ }^{1}$ H. Kroemer and M. Thomas, Superlattices Microstruct. 21, 61 (1997)

${ }^{2}$ A. F. Volkov and A. V. Zaitsev, Phys. Rev. B 53, 9267 (1996).

${ }^{3}$ N. R. Claughton, M. Leadbeater, and C. J. Lambert, J. Phys.: Condens. Matter 7, 8757 (1995).

${ }^{4}$ A. W. Kleinsasser et al., Phys. Rev. Lett. 72, 1738 (1994).

${ }^{5}$ N. van der Post, E. T. Peters, I. K. Yanson, and J. M. van Ruitenbeek, Phys. Rev. Lett. 73, 2611 (1994).

${ }^{6}$ Y. Takagaki and H. Takayanagi, Phys. Rev. B 53, 14530 (1996).

${ }^{7}$ A. L. Yeyati, A Martin-Rodero, and J. C. Cuevas, J. Phys.: Condens. Matter 8, 449 (1996).

${ }^{8}$ H. Takayanagi, T. Akazaki, and J. Nitta, Phys. Rev. Lett. 75, 3533 (1995).

${ }^{9}$ J. C. Cuevas, A. Martin-Rodero, and A. L. Yeyati, Phys. Rev. B 54, 7366 (1996).

${ }^{10}$ A. Kastalsky et al., Phys. Rev. Lett. 67, 3026 (1991).

${ }^{11}$ A. W. Kleinasser and A. Kastalsky, Phys. Rev. B 47, 8361 (1993).

${ }^{12}$ S. J. M. Bakker et al., Phys. Rev. B 48, 4168 (1993).

${ }^{13}$ M. T. Tuominen et al., Phys. Rev. Lett. 69, 1997 (1992); Phys. Rev. B 47, 11599 (1993).

${ }^{14}$ T. M. Eiles, J. M. Martinis, and M. H. Devoret, Phys. Rev. Lett. 70, 1862 (1993).

${ }^{15}$ F. W. J. Hekking et al., Phys. Rev. Lett. 70, 4138 (1993).

${ }^{16}$ J. M. Hergenrother, M. T. Tuominen, and M. Tinkham, Phys. Rev. Lett. 72, 1742 (1994).
${ }^{17}$ A. Amar et al., Phys. Rev. Lett. 72, 3234 (1994).

${ }^{18}$ U. Hanke, Yu. Galperin, K. A. Chao, M. Gisselfält, M. Jonson, and R. I. Shektes, Phys. Rev. B 51, 9084 (1995).

${ }^{19}$ D. C. Ralph, C. T. Black, and M. Tinkham, Phys. Rev. Lett. 74, 3241 (1995).

${ }^{20}$ A. L. Yeyati et al., Phys. Rev. B 55, R6137 (1997).

${ }^{21}$ S. Ishizaka, J. Sone, and T. Ando, Phys. Rev. B 52, 8358 (1995).

${ }^{22}$ C. W. J. Beenakker, Phys. Rev. B 46, 12841 (1992).

${ }^{23}$ R. Fazio and R. Raimondi, Phys. Rev. Lett. 80, 2913 (1998).

${ }^{24}$ For discussions of gauge invariance, see Büttiker and T. Christen, in Mesoscopic Electron Transport, NATO Advanced Study Institute, Series E: Applied Science, edited by L. L. Sohn, L. P. Kouwenhoven, and G. Schoen (Kluwer Academic, Dordrecht, 1997), Vol. 345, p. 259.

${ }^{25}$ G. E. Blonder, M. Tinkham, and T. M. Klapwijk, Phys. Rev. B 25, 4515 (1982)

${ }^{26}$ A.-P. Jauho, N. S. Wingreen, and Y. Meir, Phys. Rev. B 50, 5528 (1994).

${ }^{27}$ Qing-feng Sun and Tsung-han Lin, J. Phys.: Condens. Matter 9, 4875 (1997).

${ }^{28}$ A. L. Yeyati, A. Martin-Rodero, and F. J. Garcia-Vidal, Phys. Rev. B 51, 3743 (1995).

${ }^{29}$ N. S. Wingreen, K. W. Jacobsen, and J. W. Wilkins, Phys. Rev. B 40, 11834 (1989).

${ }^{30}$ For the discussion of gauge invariance for N-QD-N systems in the presence of the long-range Coulomb interaction, see Ref. 24 and Z. S. Ma, J. Wang, and H. Guo, Phys. Rev. B 57, 9108 (1998). 